\title{
A measure of distance between judgment sets
}

\author{
Conal Duddy • Ashley Piggins
}

Received: 7 October 2010 / Accepted: 11 July 2011 / Published online: 29 July 2011

(C) Springer-Verlag 2011

\begin{abstract}
In the literature on judgment aggregation, an important open question is how to measure the distance between any two judgment sets. This is relevant for issues of social choice: if two individuals hold different beliefs then we might want to find a compromise that lies somewhere between them. We propose a set of axioms that determine a measure of distance uniquely. This measure differs from the widely used Hamming metric. The difference between Hamming's metric and ours boils down to one axiom. Given judgment sets $A$ and $B$, this axiom says that if the propositions in $A \cap B$ jointly imply that the propositions in $A-B$ share the same truth value, then the disagreement between $A$ and $B$ over those propositions in $A-B$ should be counted as a single disagreement. We consider the application of our metric to judgment aggregation, and also use the metric to measure the distance between preference rankings.
\end{abstract}

\section{Introduction}

A large literature on judgment aggregation exists, motivated by List and Pettit (2002) initial contribution. ${ }^{1}$ The literature is concerned with aggregating profiles of individual judgment sets into a collective judgment set. What is a 'judgment' set? First, consider a set of propositions that is finite and closed under negation (that is, if a

\footnotetext{
1 List and Puppe (2009) is a survey to which we refer the interested reader. See also List and Polak (2010) which is an introduction to the symposium in JET on this topic.
}

C. Duddy

Government of Ireland Scholar, J.E. Cairnes School of Business and Economics,

National University of Ireland Galway, University Road, Galway, Ireland

e-mail: conal.duddy@nuigalway.ie

\section{A. Piggins $(\varangle)$}

J.E. Cairnes School of Business and Economics, National University of Ireland Galway,

University Road, Galway, Ireland

e-mail: ashley.piggins@ @uigalway.ie 
proposition is contained in the set then so is its negation). This set is called the agenda. A (individual) judgment set is a subset of the agenda, containing those propositions that the individual believes to be true. A judgment set is complete if it contains one of every proposition/negation pair in the agenda. In this article, we assume that all judgment sets are complete. Consider the agenda $\{p, \neg p, q, \neg q, p \wedge q, \neg(p \wedge q)\}$. Since these propositions are logically interconnected, a judgment set must contain $p \wedge q$ if it contains both $p$ and $q$. Another must contain $\neg(p \wedge q)$ if it contains both $p$ and $\neg q$ and so on. These are requirements of logical consistency, and we assume that judgment sets are consistent in this sense.

A central concern in the literature on judgment aggregation is that, for some agendas, this requirement of logical consistency is not compatible with the majoritarian approach to aggregation. This is illustrated by Kornhauser and Sager's (1993) celebrated example. $^{2}$

Suppose that there are three judges who must collectively decide on a case of breach of contract. The legal doctrine, which each judge accepts, states that the defendant owes compensation to the plaintiff (proposition $r$ ) if and only if the contract between them is valid (proposition $p$ ) and the defendant broke it (proposition $q$ ). Suppose that the judges hold the following beliefs.

As we see, majority voting on each proposition results in the inconsistent collective judgment set $\{p, q, r \leftrightarrow(p \wedge q), \neg r\}$. Just as the majority voting paradox can be generalised through Arrow's (1963) impossibility theorem, so can this "doctrinal paradox". List and Pettit (2002) arrived at the first systematic result in this area by showing that no aggregation rule applied to an agenda containing certain propositions can satisfy four conditions. More general results have been obtained by Dietrich (2006, 2007), Dietrich and List (2009, 2010), Dokow and Holzman (2010) and Nehring and Puppe (2010) amongst others. ${ }^{3}$

A condition that has been central in the literature, particularly when deriving impossibility theorems, is the 'independence' condition. This is the analogue of the familiar 'independence of irrelevant alternatives' condition from the preference aggregation literature. This condition says that the collective judgment on any proposition $p$ within the agenda should depend only on individual judgments on $p$. To illustrate, majority voting on each proposition, as in Table 1, satisfies this condition.

The problem with the independence condition is that it cannot be reconciled with a commitment to democratic and collectively rational aggregation. To be more precise, if the agenda satisfies certain combinatorial properties, and we want unanimous judgments to be respected at the collective level, then any independent aggregation rule is either dictatorial or produces logically inconsistent collective outcomes. ${ }^{4}$ Neither of these properties is desirable and so independence must be rejected. ${ }^{5}$

\footnotetext{
2 See also Kornhauser (1992).

3 A number of extensions of the basic judgment aggregation model have been proposed, most of which are discussed in List and Puppe (2009) and List and Polak (2010).

4 This is the central theorem in the literature. See Dokow and Holzman (2010), Dietrich and List (2007) and Nehring and Puppe (2002).

5 In Duddy and Piggins (2010), we show how rejecting the independence condition can lead to a counterpart of the Borda rule in a 'possible worlds' model of judgment aggregation. This approach began with
} 
Table 1 The doctrinal paradox

\begin{tabular}{lcclc}
\hline & $p$ & $q$ & $r \leftrightarrow(p \wedge q)$ & $r$ \\
\hline Judge 1 & $\mathrm{T}$ & $\mathrm{T}$ & $\mathrm{T}$ & $\mathrm{T}$ \\
Judge 2 & $\mathrm{F}$ & $\mathrm{T}$ & $\mathrm{T}$ & $\mathrm{F}$ \\
Judge 3 & $\mathrm{T}$ & $\mathrm{F}$ & $\mathrm{T}$ & $\mathrm{F}$ \\
Majority judgment & T & T & T & F \\
\hline
\end{tabular}

A very natural approach to the problem of judgment aggregation, other than the majoritarian one, is to determine the 'geometric median' of the profile of individual judgment sets. That is, to view the problem of judgment aggregation as one of distance minimisation. ${ }^{6}$ This is an approach that rejects independence when the propositions in the agenda are logically interconnected, as we will see in Sect. 3.1.

Distance-based rules were originally introduced in the area of belief merging in computer science. ${ }^{7}$ A distance-based rule maps each profile of individual judgment sets $\left(A_{1}, \ldots, A_{n}\right)$ to a consistent and complete collective judgment set $A$ that minimises the total distance from the individual judgment sets, i.e., selects an $A$ such that $\sum_{i \in N} d\left(A, A_{i}\right)$ is minimised. ${ }^{8}$ Distance-based rules capture the idea of reaching the best compromise between different individuals' judgment sets. Miller and Osherson (2009) discuss other kinds of distance-based procedures in addition to this, but the one above is the most natural.

How should we measure the distance between any two individuals' judgment sets? This is a central question for distance-based judgment aggregation.

The most widely used metric in the literature is the Hamming metric. This is simply the number of propositions over which the two individuals disagree. So the distance between $\{p, q, p \wedge q\}$ and $\{p, \neg q, \neg(p \wedge q)\}$ is 2 . But therein lies the problem. The proposition $\neg(p \wedge q)$ is a logical consequence of $p$ and $\neg q$, and $p \wedge q$ is a logical consequence of $p$ and $q$. So, given that the individuals both accept $p$, the disagreement over $p \wedge q$ is implied by the disagreement over $q$. The Hamming metric appears to be double counting because it ignores the fact that the propositions are logically interconnected.

In this article, we propose a set of axioms that determine an alternative metric uniquely. This metric avoids the problem identified in the previous example. The metric is based around the idea of 'betweenness'. Judgment set $C$ is between judgment sets $A$ and $B$ if $A, B$ and $C$ are distinct and, on each proposition, $C$ agrees with $A$ or with $B$ (or both). This concept of betweenness is similar to one used by Kemeny

\footnotetext{
Footnote 5 continued

List (2008). It is worth pointing out that for a certain family of many-valued models, independence is compatible with unanimity, non-dictatorship and a deductive closure condition. See Duddy and Piggins (2009).

6 Pigozzi (2006) and Miller and Osherson (2009) discuss the distance-based approach to judgment aggregation.

7 Konieczny and Pino Pérez (2002).

${ }^{8} N$ is the set of individuals and $d$ is the metric.
} 


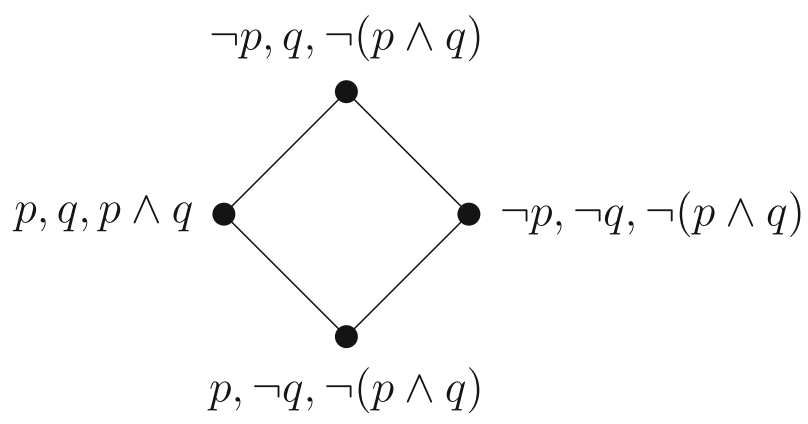

Fig. 1 Graph with judgment sets as vertices

and Snell in their characterisation of the well-known Kemeny measure of distance between preference rankings. ${ }^{9}$

We now explain our metric. Imagine a graph where each feasible judgment set is a vertex. We join two judgment sets with an edge if there is no other judgment set between them. The distance between two judgment sets is the length of the shortest path from one to the other.

Therefore, the distance from $\{p, \neg q, \neg(p \wedge q)\}$ to $\{p, q, p \wedge q\}$ is just 1 , even though they differ on 2 propositions. The sets $\{p, \neg q, \neg(p \wedge q)\}$ and $\{\neg p, q, \neg(p \wedge q)\}$ also differ on 2 propositions, but the distance between them is 2 .

The graph in this example is represented in Fig. 1.

Here is another way of thinking about our metric. To switch from $\{p, q, p \wedge q\}$ to $\{p, \neg q, \neg(p \wedge q)\}$ requires just one change in belief ( $q$ to $\neg q$, for instance). The distance between two judgment sets is the smallest number of logically coherent changes needed to convert one into the other.

The classic 'doctrinal paradox' discussed above provides a more complex example. We will refer again to this example later in the article to help clarify one of our axioms. Here the agenda is $\{p, \neg p, q, \neg q, r, \neg r,((p \wedge q) \leftrightarrow r), \neg((p \wedge q) \leftrightarrow r)\}$. The corresponding graph is represented in Fig. 2.

In Fig. 2, $A=\{p, q, r,(p \wedge q) \leftrightarrow r\}, B=\{p, \neg q, \neg r,(p \wedge q) \leftrightarrow r\}, C=$ $\{\neg p, \neg q, \neg r,(p \wedge q) \leftrightarrow r\}$ and $D=\{\neg p, q, \neg r,(p \wedge q) \leftrightarrow r\}$. Like before, we connect two judgment sets with an edge if there is no judgment set between them. As we can see, no judgment set is more than a distance of 2 away from any other.

It is worth pointing out that the Hamming distance between two judgment sets can also be calculated by determining the length of a shortest path in a graph. To see this, note that our notion of betweenness can be applied to all complete judgment sets, not just consistent ones. If we admit these inconsistent judgment sets, then (returning to our original example) the graph is as illustrated in Fig. 3.

The Hamming distance between $\{p, \neg q, \neg(p \wedge q)\}$ and $\{p, q, p \wedge q\}$ is 2 , the length of the shortest path between them.

Here is an outline of this article. Section 2 contains an axiomatisation of our metric. In Sect. 3.1, we consider the application of our metric to distance-based judgment

9 See Kemeny and Snell (1962, Chap. 2). We discuss Kemeny and Snell's contribution in Sect. 3.2. 


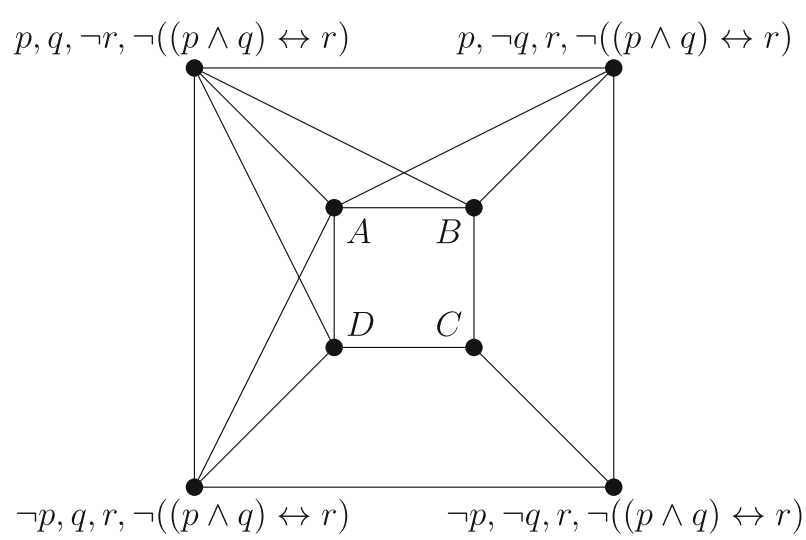

Fig. 2 Graph for the doctrinal paradox agenda

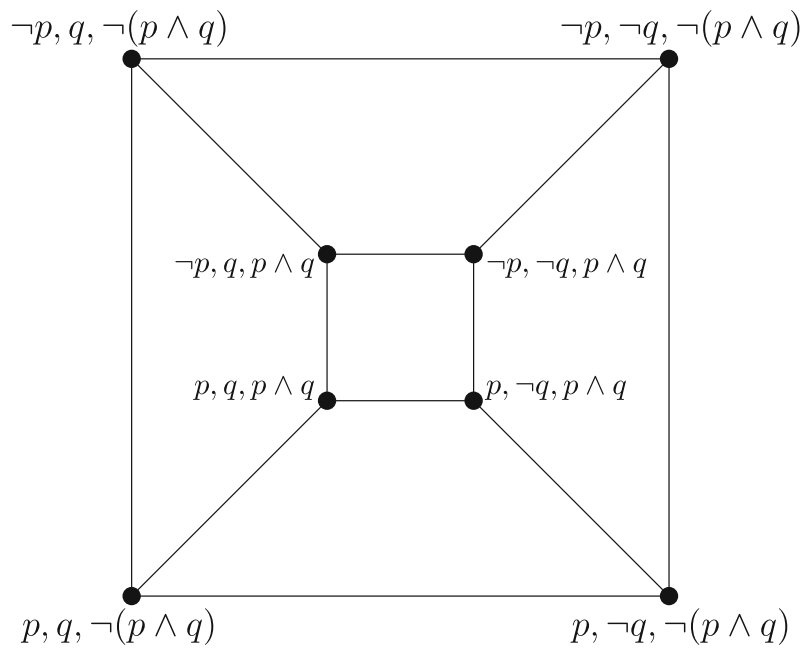

Fig. 3 Graph with all complete judgment sets as vertices

aggregation. We show, using the natural distance-based procedure described earlier, that our metric can produce a different collective outcome than that which would arise under Hamming's metric. The two metrics differ then in their implication. We also show that our metric can produce a different collective outcome than that which would arise under a 'premiss-based' approach to judgment aggregation. In Sect. 3.2, we use the metric to measure the distance between preference rankings. The final section concludes.

\section{Axiomatisation}

In this section, we provide an axiomatisation of our metric. 
For any given agenda, let $\mathcal{F}$ be the set of all complete and consistent judgment sets. A measure of distance between judgment sets $A$ and $B$ will be denoted by $d(A, B)$, where $d: \mathcal{F} \times \mathcal{F} \rightarrow \mathbb{R}$.

The first three axioms say that $d$ is a metric.

Axiom $1 d(A, B)=0$ if and only if $A=B$.

Axiom $2 d(A, B)=d(B, A)$.

Axiom $3 d(A, B) \leq d(A, C)+d(C, B)$.

For all $A, B, C \in \mathcal{F}$, we say that $C$ is between $A$ and $B$ if and only if $A \neq C \neq B$ and $(A \cap B) \subset C$. We use the word 'between' here not in the geometric sense but rather to mean that $C$ represents a compromise between $A$ and $B$.

Axiom 4 If there is a judgment set between $A$ and $B$ then there exists $C \in \mathcal{F}-\{A, B\}$ such that $d(A, B)=d(A, C)+d(C, B)$.

Axiom 4 requires that the distance function should at least partially reflect the concept of betweenness defined above. The axiom states that when there is a judgment set between $A$ and $B$ according to that definition, then there should also be a judgment set between them in the geometric sense. We do not go so far as to make the additional requirement that the judgment sets that are between $A$ and $B$ in the former sense are the same as the judgment sets that are between $A$ and $B$ in the latter sense.

It is important to note that the Hamming metric satisfies Axiom 4. Moreover, that additional requirement, that the two concepts of betweenness coincide, is also satisfied by that measure of distance for any given agenda. This is not true of our metric. ${ }^{10}$

The critical axiom is Axiom 5.

Axiom 5 If there is no judgment set between $A$ and $B$, with $A \neq B$, then $d(A, B)=1$. Hamming's metric violates Axiom 5. No judgment set exists between $\{p, q, p \wedge q\}$ and $\{p, \neg q, \neg(p \wedge q)\}$ yet the Hamming distance between them is 2 .

Here is our justification for Axiom 5. Take two distinct judgment sets $A$ and $B$ that have no judgment set between them. This means that there is no judgment set in $\mathcal{F}-\{A, B\}$ that is a superset of $A \cap B$. Therefore, if one accepts the propositions in $A \cap B$ then one must either accept every proposition in $A-B$ or reject every proposition in $A-B$. In other words, if the propositions in $A \cap B$ are true, then the propositions in $A-B$ are logically equivalent. Since $A$ and $B$ both accept the propositions in $A \cap B$, the disagreement between $A$ and $B$ over the propositions in $A-B$ should simply count as one disagreement. Hence, $d(A, B)=1$.

The argument above is perfectly general, but the examples below may help clarify it.

Example 1 The agenda is $\{p, \neg p, q, \neg q, p \wedge q, \neg(p \wedge q)\}$ (see Fig. 1). Let $A=$ $\{p, q, p \wedge q\}$ and $B=\{p, \neg q, \neg(p \wedge q)\} . A \cap B=\{p\}$ and there is no judgment set in $\mathcal{F}-\{A, B\}$ that is a superset of $A \cap B$. Note that $A-B=\{q, p \wedge q\}$. Consider the following truth table.

\footnotetext{
10 For example, consider the judgment sets $A=\{\neg p, q, \neg(p \wedge q)\}, B=\{p, \neg q, \neg(p \wedge q)\}$ and $C=$ $\{p, q, p \wedge q\}$. We can see that $C$ is not between $A$ and $B$ in the sense of being a compromise. Under the Hamming metric $C$ is also not between $A$ and $B$ in the geometric sense. That is, using $h$ to denote the Hamming metric, we have $h(A, B)<h(A, C)+h(C, B)$. Under the Hamming metric the two concepts of betweenness will always agree in this way. However, under our metric $C$ is between $A$ and $B$ in the geometric sense. That is, using $d$ to denote our metric, we have $d(A, B)=d(A, C)+d(C, B)$ (see Fig. 1).
} 


\begin{tabular}{ccc}
\hline$p$ & $q$ & $p \wedge q$ \\
\hline $\mathrm{T}$ & $\mathrm{T}$ & $\mathrm{T}$ \\
$\mathrm{T}$ & $\mathrm{F}$ & $\mathrm{F}$ \\
\hline
\end{tabular}

Both $A$ and $B$ agree that $p$ is true. Given this, there are only two possibilities. Either you accept both $q$ and $p \wedge q$ or you reject both $q$ and $p \wedge q$ (and accept $\neg q$ and $\neg(p \wedge q))$. Judgment set $A$ takes the former route, judgment set $B$ takes the latter route. However, notice from the truth table that from the perspective of proposition $p$ (which both $A$ and $B$ agree is true), the propositions $q$ and $p \wedge q$ are logically equivalent. Given this, the different truth values assigned to these propositions by $A$ and $B$ should count as reflecting just one disagreement, and so $d(A, B)$ should be one. Hamming's metric counts them as reflecting two disagreements.

Example 2 The agenda is $\{p, \neg p, q, \neg q, r, \neg r,((p \wedge q) \leftrightarrow r), \neg((p \wedge q) \leftrightarrow r)\}$ (see Fig. 2). Let $A=\{p, q, \neg r, \neg((p \wedge q) \leftrightarrow r)\}$ and $B=\{\neg p, q, \neg r,((p \wedge q) \leftrightarrow$ $r)\}$. $A \cap B=\{q, \neg r\}$ and there is no judgment set in $\mathcal{F}-\{A, B\}$ that is a superset of $A \cap B$. Note that $A-B=\{p, \neg((p \wedge q) \leftrightarrow r)\}$. Consider the following truth table.

\begin{tabular}{cccc}
\hline$q$ & $\neg r$ & $p$ & $\neg((p \wedge q) \leftrightarrow r)$ \\
\hline $\mathrm{T}$ & $\mathrm{T}$ & $\mathrm{T}$ & $\mathrm{T}$ \\
$\mathrm{T}$ & $\mathrm{T}$ & $\mathrm{F}$ & $\mathrm{F}$ \\
\hline
\end{tabular}

Both $A$ and $B$ accept $q$ and $\neg r$. Given this, there are only two possibilities. Either you accept both $p$ and $\neg((p \wedge q) \leftrightarrow r)$ or you reject both $p$ and $\neg((p \wedge q) \leftrightarrow r)$ (and accept $\neg p$ and $((p \wedge q) \leftrightarrow r))$. Judgment set $A$ takes the former route, judgment set $B$ takes the latter route. However, notice from the truth table that from the perspective of the set of propositions $\{q, \neg r\}$ (which both $A$ and $B$ accept), the propositions $p$ and $\neg((p \wedge q) \leftrightarrow r)$ are logically equivalent. Given this, the different truth values assigned to these propositions by $A$ and $B$ should count as reflecting just one disagreement, and so $d(A, B)$ should be one. Hamming's metric counts them as reflecting two disagreements.

These examples help clarify our argument for Axiom 5.

We now present our theorem.

Let $\mathcal{G}=(\mathcal{V}, \mathcal{E})$ be a graph with the same number of vertices as there are judgment sets in $\mathcal{F}$, and a set of edges $\mathcal{E}$ defined as follows. Let $v$ be a bijection from $\mathcal{F}$ to the set of vertices $\mathcal{V}$. For all $A, B \in \mathcal{F}$, the set of edges $\mathcal{E}$ contains $\{v(A), v(B)\}$ if and only if there is no judgment set between $A$ and $B$.

Define a function $g$ with domain $\mathcal{F} \times \mathcal{F}$ as follows. For all $A, B \in \mathcal{F}, g(A, B)$ is equal to the length of a shortest path from $v(A)$ to $v(B)$ in graph $\mathcal{G}$.

Our theorem is the following. ${ }^{11}$

Theorem The function $g$ is unique in satisfying all of the axioms.

Proof We first prove that $g$ satisfies Axioms 1-5. It suffices to check that the graph $\mathcal{G}$ is connected. This means that it is possible to find a path from any vertex to any other vertex of $\mathcal{G}{ }^{12}$ Let us note here that, for all $A, B, C, D \in \mathcal{F}$, (i) if $C$ is between $A$ and $B$

\footnotetext{
11 We are grateful to a referee for suggesting a shortening of our original proof.

12 Our $g$ function is the standard concept of distance in graph theory, commonly known as the geodesic distance. If $\mathcal{G}$ is connected then we can be sure that $g$ satisfies all of our axioms.
} 
then $B$ is not between $A$ and $C$ and (ii) if $C$ is between $A$ and $B$, but $D$ is not between $A$ and $B$ then $D$ is not between $A$ and $C$. These two statement are easy to verify. Now let us suppose that there exist $A, B \in \mathcal{F}$ such that there is no path from $v(A)$ to $v(B)$ in graph $\mathcal{G}$. It follows that $\{v(A), v(B)\}$ is not contained in the set of edges $\mathcal{E}$. Recalling the definition of $\mathcal{G}$, we see that there must exist $C \in \mathcal{F}-\{A, B\}$ such that $C$ is between $A$ and $B$. Of course, as there is no path connecting $v(A)$ and $v(B), v(C)$ cannot be connected to both $v(A)$ and $v(B)$. Assume without loss of generality that $v(A)$ and $v(C)$ are not connected. So there must exist $D \in \mathcal{F}-\{A, C\}$ such that $D$ is between $A$ and $C$, and $v(D)$ cannot be connected to both $v(A)$ and $v(C)$. It is implied by (i) and (ii) above that $D \in \mathcal{F}-\{A, B, C\}$. By repeatedly applying this type of argument, we see that the set $\mathcal{F}$ must be infinite. This is a contradiction. So we conclude that $\mathcal{G}$ is connected.

We now prove that no other metric satisfies all of the axioms. Let $d$ be a metric satisfying all of the axioms. We claim that $d=g$.

By construction, $g(A, B)=1$ implies that there is no judgment set between $A$ and $B$. So, by Axiom 5, if $n=1$ then $g(A, B)=n$ implies $d(A, B)=n$. We proceed by induction on $n$. Fix any $n \in \mathbb{N}$ and assume that, for all $k \in\{1, \ldots, n\}$ and all $A, B \in \mathcal{F}$, if $g(A, B)=k$ then $d(A, B)=k$. Take two judgment sets $A, B \in \mathcal{F}$ with $g(A, B)=n+1$. Consider any judgment set $C$ that is on a shortest path from $A$ to $B$ in graph $\mathcal{G}$. Since $g(A, C)$ and $g(C, B)$ are in $\{1,2, \ldots, n\}$, we know that $d(A, C)=$ $g(A, C)$ and $d(C, B)=g(C, B)$. It follows that $d(A, C)+d(C, B)=n+1$ and so $d(A, B) \leq n+1$.

By Axiom 4, there is a judgment set $D$ such that $d(A, B)=d(A, D)+d(D, B)$. If the path from $A$ to $D$ is greater than $n$ steps then there must be an $E$ such that $d(A, D)=d(A, E)+d(E, D)$. In this way, we can construct a sequence such that $d(A, B)=d\left(A, A_{1}\right)+d\left(A_{1}, A_{2}\right)+\cdots+d\left(A_{m}, B\right)$, with each segment on the righthand side requiring no more than $n$ steps in the graph $\mathcal{G}$. In other words, $g\left(A, A_{1}\right) \leq$ $n, g\left(A_{1}, A_{2}\right) \leq n$ and so on. By assumption then, for each of the segments, the distance given by $d$ is the same as that given by $g$. The sum of these distances must be at least $n+1$ since this is the length of the shortest path from $A$ to $B$. Hence, $d(A, B) \geq n+1$. Thus, $d(A, B)=n+1$.

\section{Applications}

\subsection{Judgment aggregation}

As discussed in Sect. 1, majority voting is not an acceptable method of aggregation for every agenda. More to the point, for many agendas all methods of aggregation that consider each proposition independently of the others are unsuitable. ${ }^{13}$ Various judgment aggregation rules exist that give up the independence condition and it may be appropriate to use these rules for some or all of these agendas. They may even be appropriate when the independence condition could be satisfied (for example, at

\footnotetext{
13 By this we mean that, for these agendas, any independent rule will either produce logically inconsistent collective outcomes, violate unanimity or be a dictatorship. See footnote 4.
} 
Table 2 Different outcome to Hamming's

\begin{tabular}{llll}
\hline & $p$ & $q$ & $p \wedge q$ \\
\hline Two individuals & $\mathrm{T}$ & $\mathrm{T}$ & $\mathrm{T}$ \\
Two individuals & $\mathrm{T}$ & $\mathrm{F}$ & $\mathrm{F}$ \\
Three individuals & $\mathrm{F}$ & $\mathrm{T}$ & $\mathrm{F}$ \\
Majority judgment & $\mathrm{T}$ & $\mathrm{T}$ & $\mathbf{F}$ \\
Our metric & $\mathrm{T}$ & $\mathrm{T}$ & $\mathrm{T}$ \\
Hamming's metric & $\mathbf{F}$ & $\mathrm{T}$ & $\mathbf{F}$ \\
Premiss-based rule & T & T & T \\
\hline
\end{tabular}

agendas where majority voting would not lead to a violation of logical consistency). We consider two rules that abandon independence in this article: distance-based rules, as described in Sect. 1 and premiss-based rules.

Premiss-based rules designate a subset of the agenda as a set of premisses and aggregate judgments on them by some independent rule, but allow the collective judgments on all other propositions (the non-premisses or 'conclusions') to depend on the resulting collective judgments on the premisses. In this way, logical consistency is 'forced' on the collective judgment set by the collective judgments reached on the premisses. For example, in Table 1, if the premisses are $\{p, q, r \leftrightarrow(p \wedge q)\}$ and we use majority voting on these, then we end up with the consistent collective judgment set $\{p, q, r \leftrightarrow(p \wedge q), r\} .^{14}$

As mentioned in Sect. 1 distance-based rules require a metric. In this section, we consider some applications of our metric. We accomplish two things. First, we demonstrate that our metric can lead to a different collective outcome than that which would arise under Hamming's metric. Second, we demonstrate that our metric can lead to a different collective outcome than that which would arise under a premiss-based approach to judgment aggregation.

Consider the agenda $\{p, \neg p, q, \neg q,(p \wedge q), \neg(p \wedge q)\}$. Consider the following profile. The graph corresponding to this agenda is given in Fig. 1.

In Table 2, we see that our metric and Hamming's metric can generate different collective outcomes from the same profile. Premiss-based aggregation (under the natural assumption that the set of premisses is $\{p, q\}$ ) generates the same outcome at this profile as our metric does. In fact, these two procedures (distance-based aggregation with our metric and premiss-based aggregation) will generate the same outcome at every profile for this agenda.

However, premiss-based aggregation and distance-based aggregation with our metric can differ at other agendas. Consider the agenda $\{p, \neg p, q, \neg q, p \leftrightarrow q, \neg(p \leftrightarrow$ $q)\}$. Consider the profile in Table 3. The graph corresponding to this agenda is given in Fig. 4.

In Table 3, we see that the distance-based approach with our metric generates a different collective outcome than that which would arise under the premiss-based approach (again, under the natural assumption that the set of premisses is $\{p, q\}$ ).

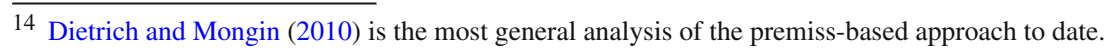


Table 3 A different outcome than the premiss-based approach

\begin{tabular}{llll}
\hline & $p$ & $q$ & $p \leftrightarrow q$ \\
\hline Two individuals & $\mathrm{T}$ & $\mathrm{T}$ & $\mathrm{T}$ \\
Two individuals & $\mathrm{T}$ & $\mathrm{F}$ & $\mathrm{F}$ \\
Three individuals & $\mathrm{F}$ & $\mathrm{T}$ & $\mathrm{F}$ \\
Our metric & $\mathrm{F}$ & $\mathbf{T}$ & $\mathbf{F}$ \\
Premiss-based & $\mathrm{T}$ & $\mathbf{T}$ & $\mathrm{T}$ \\
\hline
\end{tabular}

$\neg p, q, \neg(p \leftrightarrow q)$

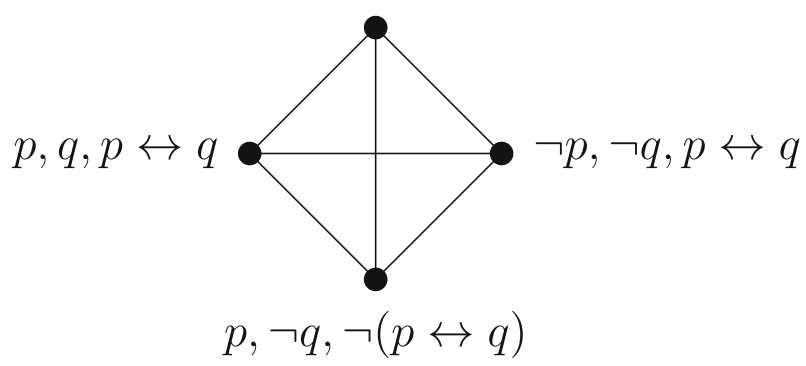

Fig. 4 Graph for agenda $\{p, \neg p, q, \neg q, p \leftrightarrow q, \neg(p \leftrightarrow q)\}$

So it is not evident that distance-based judgment aggregation under our metric is biased towards premiss-based aggregation in general.

One property of the distance-based approach is that the judgment set that minimises the total distance from the individual judgment sets may not be unique. For example, with the agenda $\{p, \neg p, q, \neg q,(p \wedge q), \neg(p \wedge q)\}$ if each complete and consistent judgment set is held by just one person, then the distance-based approach produces a 'tie'. In this case, every judgment set will minimise the total distance. Returning to the legal origins of the theory, we can interpret this as a situation in which a court is unable to reach a verdict. Notice, however, that a tie will not arise in the case of the classic doctrinal paradox (see Table 1). In that case, the collective judgment set is unique and corresponds to $\{p, q, r \leftrightarrow(p \wedge q), r\}$.

\subsection{Preference rankings}

Kemeny (1959) introduced a measure of distance between preference rankings that has become standard in the social choice literature. Here, we model a preference ranking by the asymmetric part of a weak ordering. ${ }^{15}$ While the Hamming distance between judgment sets $A$ and $B$ is $|A-B|$, the Kemeny distance between preference rankings $P$ and $P^{\prime}$ is $\left|P-P^{\prime}\right|+\left|P^{\prime}-P\right|$. We can immediately see that the Kemeny distance is essentially the application of Hamming distance to preference rankings. The addition of $\left|P^{\prime}-P\right|$ is required because relations and judgment sets represent

\footnotetext{
15 In other words, we model a preference ranking by a binary relation that is asymmetric and negatively transitive. We interpret incompleteness as indifference.
} 
negation differently. The negation of $x P y$ is represented by the absence of $(x, y)$ from $P$, whereas the negation of a proposition $p$ is represented by the inclusion of $\neg p$ in $A$ (as well as the exclusion of $p$ ).

Kemeny and Snell (1962) present an axiomatisation of the Kemeny metric. We have followed their approach in this article. They define 'betweenness' in the same way that we do, although they use the word in the inclusive sense. For Kemeny and Snell, $P^{\prime}$ is between $P$ and $P^{\prime \prime}$ if and only if $P \cap P^{\prime \prime} \subseteq P^{\prime} \subseteq P \cup P^{\prime \prime}$. It is equivalent to say $P^{\prime}$ is between $P$ and $P^{\prime \prime}$ if and only if $J(P) \cap J\left(P^{\prime \prime}\right) \subseteq J\left(P^{\prime}\right)$, where $J(P)$ is the judgment set counterpart of $P$ (which we will be define below).

We outline here the six axioms used by Kemeny and Snell (K-S). Our Axioms 1 and 2 are the same as the first and second $\mathrm{K}-\mathrm{S}$ axioms. The third $\mathrm{K}-\mathrm{S}$ axiom combines our Axiom 3 with the requirement that $d\left(P, P^{\prime \prime}\right)=d\left(P, P^{\prime}\right)+d\left(P^{\prime}, P^{\prime \prime}\right)$ if and only if $P^{\prime}$ is between $P$ and $P^{\prime \prime}$ (inclusively). Note that this requirement is more demanding than our Axiom 4. The fourth $\mathrm{K}-\mathrm{S}$ axiom is a standard neutrality condition, requiring that the names of the alternatives do not matter. The fifth $\mathrm{K}-\mathrm{S}$ axiom states that if two rankings have the same top (or bottom) equivalence class then their distance can be calculated as if the items in that equivalence class were not being ranked. The final $\mathrm{K}-\mathrm{S}$ axiom fixes a unit of measurement by requiring that the minimum positive distance is 1 .

A measure of distance between judgment sets can be used to measure the distance between preference rankings provided that we translate the rankings into judgment sets. Let $T$ be a two-place predicate and $X$ a finite set of alternatives. Construct an agenda containing, for all $x, y \in X$, the propositions $x T y$ and $\neg x T y$. Given a binary relation $P$ over $X$, let us define its judgment set counterpart $J(P)$ as follows. For all $x, y \in X$, if $x P y$ then $x T y \in J(P)$, otherwise $\neg x T y \in J(P)$. The set of feasible judgment sets contains all and only those judgment sets that correspond to preference rankings over $X$. We can now see that the Kemeny distance from $P$ to $P^{\prime}$ is equal to the Hamming distance from $J(P)$ to $J\left(P^{\prime}\right)$.

We now show how our metric can be used to measure the distance between preference rankings. Abusing notation, we write $g\left(P, P^{\prime}\right)$ instead of $g\left(J(P), J\left(P^{\prime}\right)\right)$ in what follows. An important feature of our metric is that when there is no judgment set between $A$ and $B$ the distance from $A$ to $B$ is 1 . This means that our metric, when applied to preference rankings, must be different to Kemeny's. To see this, suppose that $P$ and $P^{\prime}$ are preference rankings over $\{x, y, z\}$, with $P$ ranking $x$ first, and $y$ and $z$ tied second, whilst $P^{\prime}$ places all three items in the same equivalence class. The Kemeny distance is 2 since $P^{\prime}$ contains $(y, x)$ and $(z, x)$ while $P$ excludes both of these. However, there is no preference ranking between $P$ and $P^{\prime}$ (exclusively).

Let us further illustrate the difference between the Kemeny metric and our metric. Consider the relations $P=\{(x, y),(y, z),(x, z)\}$ and $P^{\prime}=\{(y, x),(z, y),(z, x)\}$. Let $k$ denote the Kemeny metric. We find that $k\left(P, P^{\prime}\right)=6$ and $g\left(P, P^{\prime}\right)=4$. One way of thinking about this distance is that the Kemeny metric $k$ counts six steps:

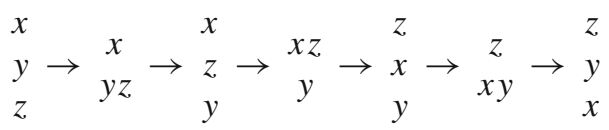


Our metric $g$ counts four steps:

$$
\begin{aligned}
& x \\
& y \rightarrow \\
& z
\end{aligned} \rightarrow x y z \rightarrow \begin{gathered}
z \\
x y
\end{gathered} \rightarrow \begin{aligned}
& z \\
& y \\
& x
\end{aligned}
$$

The distances determined by $k$ and $g$ do not always differ. Consider $P^{\prime \prime}=$ $\{(y, x),(y, z),(x, z)\}$. We find that $k\left(P, P^{\prime \prime}\right)=g\left(P, P^{\prime \prime}\right)=2$. It is also true that $k\left(P^{\prime \prime}, P^{\prime}\right)=g\left(P^{\prime \prime}, P^{\prime}\right)=4$. Indeed, this serves as an example of our metric violating the third $\mathrm{K}-\mathrm{S}$ axiom. Note that $P^{\prime \prime}$ is between $P$ and $P^{\prime}$ and yet $g\left(P, P^{\prime}\right)<g\left(P, P^{\prime \prime}\right)+g\left(P^{\prime \prime}, P^{\prime}\right)$. Our metric satisfies all of the other $\mathrm{K}-\mathrm{S}$ axioms.

Suppose that we model preferences by linear orders only. ${ }^{16}$ When indifference is not permitted it is natural to define the Kemeny distance between preference rankings $P$ and $P^{\prime}$ as being $\frac{1}{2}\left(\left|P-P^{\prime}\right|+\left|P^{\prime}-P\right|\right)$, which is equal to $\left|P-P^{\prime}\right|$. In the judgment set framework, the set of feasible judgment sets now contains only those judgment sets that correspond to linear orders. Here we find that, for all $P, P^{\prime}$, the Kemeny distance from $P$ to $P^{\prime}$ is equal to the distance from $J(P)$ to $J\left(P^{\prime}\right)$ as determined by our metric.

We can think about the difference between the Kemeny metric and our metric in the following way. Under the Kemeny metric, the path from $\{(x, y),(y, z),(x, z)\}$ to $\{(y, x),(z, y),(z, x)\}$ requires six steps because, at each step, we can raise or lower the position of one alternative relative to just one other alternative. Under our metric, at each step we can raise or lower the position of one alternative relative to multiple other alternatives, provided those other alternatives are together in a single equivalence class. This is why the restriction to linear orders eliminates the difference between the metrics.

\section{Conclusion}

We have identified a weakness with Hamming's metric when applied to judgment sets. Hamming's metric fails to respect the fact that propositions are logically interconnected, and this leads it to double count. We have proposed an alternative metric, and we have demonstrated that it is unique in satisfying five axioms. We have also explored the implications of using the metric in distance-based judgment aggregation. In addition, we have shown how the metric can be used to derive a measure of distance between preference rankings.

We hope that this article stimulates further study on metrics for judgment sets. Do better metrics exist than the one we have described here? What are the implications of these alternative metrics for judgment aggregation? In addition, can an axiomatic basis be found for the distance-based procedure itself? ${ }^{17}$ We leave these questions to future research.

\footnotetext{
16 By a linear order we mean a binary relation that is asymmetric, transitive and complete.

17 This last question would mirror what Young and Levenglick (1978) achieved for Kemeny's original social choice rule. This rule applies Kemeny's metric to the aggregation of preferences.
} 
Acknowledgments Financial support from the Irish Research Council for the Humanities and Social Sciences, the Spanish Ministry of Science and Innovation through MICINN/FEDER grant ECO2010-21624 and the NUI Galway Millennium Fund is gratefully acknowledged. We are grateful for the comments of Maurice Salles, Nick Tosh, an Associate Editor and two referees.

\section{References}

Arrow KJ (1963) Social choice and individual values. Wiley, New York

Dietrich F (2006) Judgment aggregation: (im)possibility theorems. J Econ Theory 126:286-298

Dietrich F (2007) A generalised model of judgment aggregation. Soc Choice Welf 28:529-565

Dietrich F, List C (2007) Arrow's theorem in judgment aggregation. Soc Choice Welf 29:19-33

Dietrich F, List C (2009) Propositionwise judgment aggregation: the general case. Working paper, London School of Economics

Dietrich F, List C (2010) The aggregation of propositional attitudes: towards a general theory. In: Gendler TS, Hawthorne J (eds) Oxford studies in epistemology, vol 3. Oxford University Press, Oxford

Dietrich F, Mongin P (2010) The premiss-based approach to judgment aggregation. J Econ Theory 145: $562-582$

Dokow E, Holzman R (2010) Aggregation of binary evaluations. J Econ Theory 145:495-511

Duddy C, Piggins A (2009) Many-valued judgment aggregation: characterizing the possibility/impossibility boundary for an important class of agendas. Working paper, NUI Galway

Duddy C, Piggins A (2010) Aggregating partitions. Working paper, NUI Galway

Kemeny JG (1959) Mathematics without numbers. Daedalus 88:577-591

Kemeny JG, Snell JL (1962) Mathematical models in the social sciences. Ginn, New York

Konieczny S, Pino Pérez R (2002) Merging information under constraints: a logical framework. J Log Comput 12:773-808

Kornhauser LA (1992) Modelling collegial courts II: legal doctrine. J Law Econom Organ 8:441-470

Kornhauser LA, Sager LG (1993) The one and the many: adjudication in collegial courts. Calif Law Rev $81: 1-59$

List C (2008) Which worlds are possible? A judgment aggregation problem. J Philos Log 37:57-65

List C, Pettit P (2002) Aggregating sets of judgments: an impossibility result. Econ Philos 18:89-110

List C, Polak B (2010) Introduction to judgment aggregation. J Econ Theory 145:441-446

List C, Puppe C (2009) Judgment aggregation: a survey. In: Anand P, Pattanaik P, Puppe C (eds) The handbook of rational and social choice. Oxford University Press, Oxford

Miller MK, Osherson D (2009) Methods for distance-based judgment aggregation. Soc Choice Welf 32:575-601

Nehring K, Puppe C (2002) Strategy-proof social choice on single-peaked domains: possibility, impossibility and the space between. Working paper, University of California at Davis

Nehring K, Puppe C (2010) Abstract arrowian aggregation. J Econ Theory 145:467-494

Pigozzi G (2006) Belief merging and the discursive dilemma: an argument-based account to paradoxes of judgment aggregation. Synthese 152:285-298

Young HP, Levenglick A (1978) A consistent extension of Condorcet's election principle. SIAM J Appl Math 35:285-300 